\title{
LAS COLONIAS FRONTERIZAS
}

\author{
Por \\ Jeffery Brannon*
}

\begin{abstract}
RESUMEN
Este artículo examina el rápido crecimiento de las comunidades irregulares que son habitadas por población de escasos recursos, predominantemente hispana, localizadas en áreas desincorporadas de lado norteamericano de la frontera México-Estados Unidos durante la década de los ochenta. Alrededor de 175 o $200 \mathrm{mil}$ personas actualmente viven en estas llamadas "colonias", mismas que frecuentemente están aisladas de las áreas urbanas cercanas y sin disponer de acceso adecuado a los servicios públicos básicos tales como agua, drenaje y transporte público. La inmigración de mexicanos a los Estados Unidos y la escasez de viviendas a precios módicos están identificados como los factores principales en la creación de estas comunidades. El Paso, Texas es utilizado como caso para el estudio de la dinámica social, política y económica del crecimiento de las "colonias". En este artículo se llega a la conclusión de que serán necesarias inciativas innovadoras, principalmente locales, para minimizar las consecuencias potenciales negativas del desarrollo irregular de anillos de pobreza alrededor de las áreas urbanas ubicadas en el lado norteamericano de la frontera.
\end{abstract}

\begin{abstract}
The article examines the rapid growth during the 1980 s of unregulated, low-income, and predominately hispanic communities in unincorporated areas on the U.S. side of the U.S.-Mexican border. Some 175,000-200,000 people now live in these so-called "colonias", which more often than not are isolated from nearby urban areas and without adequate access to basic public services such as water, wastewater disposal, and public transportation. Mexican immigration to the United States and a shortage of modest price housing are identified as major factors in the creation of these communities and El Paso, Texas is used as a case study of the social, political, and economic dynamics of "colonia" growth. The paper concludes that innovative, and largely local, initiatives will be needed to minimize the potential negative consequences of the unregulated development of rings of poverty around urban areas on the U.S. side of the border.
\end{abstract}

- Profesor asociado de economía en el Departamento de Economía y Finanzas de la Universidad de Texas en El Paso.

Bste artículo fue traducido del inglés por Guadalupe Ortega Villa. 


\section{INTRODUCCIÓN}

Este trabajo seffala un problema en la frontera que ha existido desde hace muchos años, sin embargo, se pensaba que sólo se presentaba en México - asentamientos no regulados y generalmente ilegales-. Estos asentamientos, frecuentemente llamados "colonias populares", pueden ser encontrados en las afueras de todas las grandes ciudades mexicanas; de hecho, este tipo de desarrollo urbano no planificado es común a todo el tercer mundo o naciones en vías de desarrollo.

En la actualidad, aproximadamente de 150,000 a 200,000 personas que habitan en la zona fronteriza norteamericana viven en asentamientos que, en cuanto a desarrollo y características, son similares a los del lado mexicano. Su crecimiento representa un reto al que temen enfrentar las autoridades locales, debido a que muchos de los residentes de estas colonias estadounidenses son de bajos ingresos, salud deficiente y sus condiciones de vida están por debajo del promedio. Casi toda la atención se ha centrado en solucionar los problemas de abastecimiento de agua potable y drenaje en las áreas afectadas; no obstante, aquí se argumenta que los problemas de agua y de drenaje son sólo síntomas, el problema real, y mucho menos sencillo, es proveer de vivienda a un gran número de habitantes hispanos de bajos ingresos. Muchos de ellos son mexicanos que optaron por migrar y asentarse en la franja fronteriza estadounidense durante la última década.

\section{¿QUÉ ES UNA COLONIA?}

Un reporte del Departamento de Servicios Humanos de Texas define las colonias como "pequeñas áreas de pobreza altamente concentrada, que están física y legalmente aisladas de las ciudades vecinas"1 (Texas Depl. of Human Services, 1988: iii); anade que se caracterizan así porque sus condiciones de vivienda, agua y drenaje son inferiores al promedio, " |a grado tal que] las condiciones de muchas colonias retan la descripción" (Texas Dept. of Human Services, 1988: iv, 1-3). Un reporte preparado in 1987 para Ronald D. Coleman, congresista texano, agrega que el limitado acceso a los servicios básicos de los residentes de las colonias es ol

1 La palabra "colonia", como es utilizada en la franja fronteriza de los Estados Unidos, $n$ inadecuada. En México, y en la mayoría de otros países latinoamericanos, no tiene unn connotación negativa, sino simplemente es un término para referirse a un barrio. Sin embarnu, debido a que el término "colonia" ahora comúnmente se usa en la frontera para descriliı desarrollos de vivienda marginal en áreas no incorporadas, en este trabajo será utilizado on ese mismo sentido. 
resultado de estar localizadas en áreas no incorporadas (Copeland and Courpas, 1987: 1). Ambas definiciones deberían corregirse para incluir el hecho de que los residentes de los colonias fronterizas estadounidenses son predominantemente de origen hispano ${ }^{2}$ (más del $90 \%$ en muchos de los casos), y que los pocos estudios que se han efectuado sugieren que muchas de las familias en las colonias tienen una o más personas recientemente emigradas de México.

Los asentamientos tipo colonia han existido en la franja fronteriza norteamericana desde hace muchos años. A finales de la década de los setenta y durante la presente, el crecimiento de estas áreas, muchas de las cuales eran ilegales, se aceleró en las afueras de ciudades como El Paso y McAllen, Texas. Las autoridades públicas locales empezaron a darse cuenta de los peligros planteados por los grandes desarrollos irregulares, pero, por motivos que después se expondrán, poco hicieron para controlarlos o elevar el nivel de los servicios públicos. No obstante, los residentes de las colonias empezaron a organizarse y a presionar a los políticos de todos los niveles para tener acceso a agua potable. Su organización fue alentada y facilitada en Texas por grupos como El Paso Interreligious Sponsoring Organization (EPISO, Organización para la Ayuda Interreligiosa de El Paso) y la Valley Interfaith in the lower Rio Grande Valley (Interfé del Valle en el valle de los bajos del río Grande). Para 1987, cuando el problema de la colonia fue expuesto al duro brillo de los medios masivos de comunicación nacional, los asentamientos fueron considerados, en palabras del jefe de planeación de la ciudad de El Paso, "como una enfermedad, un maleficio" (El Paso Herald-Post, del 7 de abril de 1987).

Como un aviso acerca de la magnitud del problema de las colonias empezaron a crecer historias en los medios de comunicación fronterizos que capturaron la atención nacional. En 1987 fueron ilustrativamente presentadas en el programa Today de la NBC, y fueron objeto de historias en revistas y periódicos como The Washington Post, Newsweek, y Life, entre otras ${ }^{3}$. Ante el incremento de la presión local para que se hiciera algo acerca del problema y la insistencia de la publicidad negativa, los políticos empezaron a buscar "a ciegas" una solución a los problemas del agua y del drenaje (U.S. Congress, 1988).

Los esfuerzos cotidianos para encontrar soluciones a la plétora de problemas que representan las colonias - para ambos, sus residentes y la

2 N. del T. El término "hispano" (hispanic) se utiliza en Estados Unidos para referirse a aquellas personas que son descendientes de mexicanos olatinoamericanos.

3 Por ejemplo, véase "El Paso Perimeter of Poverty", Washington Post, 17 de agosto de 1987 y "In Texas, A Grim New Appalachia", Newsweet, 8 de junio de 1987. pp. 27-28. 
población fronteriza en su conjunto- son constreñidos por la disposición de recursos, así como por la falta de información. Los datos del censo sun la mejor fuente de información detallada a nivel de barrio (vecindad y manzana). Sin embargo, el censo de 1980, si bien documenta esos impen tantes cambios demográficos ocurridos a lo largo de la frontera, In, previene a aquéllos que elaboran las políticas o a los investigadores acerca de la magnitud de esos cambios ocho años después.

Pocos estudios detallados y confiables han sido encomendados desde que el problema de las colonias surgió a mediados de los ochenta. Muchos de ellos han respondido a las exigencias políticas creadas por la publicidad nacional y a las presiones ejercidas por los residentes de las colonias y las organizaciones que los asisten. El reporte preparado en 1987 por cl congresista Coleman estimaba que las colonias existían en 25 de los 26 municipios fronterizos de California, Arizona, Nuevo México y Texas. II total de habitantes de las colonias fue cuantificado entre $175 \mathrm{mil}$ y 200 mil; dicha información no se obtuvo de una encuesta, sino simplemente mediante el padrón estatal y a través de autoridades locales, cuya información también era tentativa e incompleta (Copeland y Courpas, 1987). Un estudio de ingeniería solicitado por la ciudad de EI Paso reportó en 1987 que 28 mil personas de ese municipio estaban sin servicio público de agua, y que 53 mil carecían de acceso a los sistemas públicos de drenaje ( El Paso Herald-Post, 24 de noviembre de 1987); con todo, muchas de esas familias tienen pozos y fosas sépticas perfectamente seguras. ${ }^{4}$

En un reporte de 1988, el Consejo para el Desarrollo Acuífero de Texas (Texas Water Development Bord) estimó que más de 71 mil personas habitaban 435 colonias en los municipios de Cameron, Hidalgo y Willacy en los bajo del Valle Río Grande del sur de Texas, dichos asentamientos fueron descritos como "áreas residenciales ubicadas en zonas rurales caracterizadas por una alta proporción de viviendas en condiciones inferiores a los estándares, caminos y drenaje inadecuados, y deficiente servicio de agua y drenaje" (U.S. Congress, 1988: 260). Ambos estudios dicen poco acerca de los asentamientos, excepto que generalmente no tienen acceso a los servicios públicos de agua y alcantarillado. Esta parece ser la situación de la mayoría de las familias rurales estadounidenses, y ciertamente carece de fundamentos para establecer políticas públicas en una región fronteriza.

De hecho, aunque la mayoría de las familias parecen recibir ingresos que están por debajo de los promedios regionales, estatales y nacionales,

4 El autor y otras personas han confirmado este hecho mediante visita a las zonas que han sido erróneamente descritas como "colonias". 
se debe prestar mayor atención al generalizar sobre su estatus socioeconomico. En las colonias del municipio de El Paso, donde se han concentrado la mayoría de los esfuerzos de mis investigaciones, la calidad de la vivienda varfa tanto dentro de las colonias como entre ellas. Hay familias que viven en casas hechas de los materiales más fácilmente disponibles, como adobe, bloque de concreto y madera de desecho. En contraste, con frecuencia uno se encuentra en la misma colonia con casas de ladrillo bien acabado, nada diferentes de aquéllas que se podrían encontrar en los barrios de la clase obrera en la mayoría de las ciudades norteamericanas. No obstante las descripciones de la prensa popular, no son los más pobres en el municipio de El Paso; sino que son trabajadores de escasos recursos, la mayoría de los cuales no pueden encontrar vivienda para personas de bajos ingresos en la ciudad de El Paso, o quienes prefieren dejar el barrio central de la ciudad y construir una casa propia. En 1980, el $80.8 \%$ de las viviendas en las comunidades de Socorro-San Elizario estaban ocupadas por sus propietarios, en comparación con el $59.2 \%$ de la ciudad del El Paso (U.S. Census, 1980).

Los estudios comunitarios más reveladores y completos a partir de 1980, fueron llevados a cabo por el Departamento de Servicios Humanos de Texas. En abril de 1988, el departamento aplicó una encuesta a 600 familias en el municipio de El Paso y un número igual en los municipios texanos de Willacy, Cameron e Hidalgo (Texas Dept. of Human Services, 1988). La encuesta mostró claramente que mientras que estos dos grupos obtuvieron ingresos que los ubican en el quintil más bajo de las familias norteamericanas, la población de las dos áreas fue diferente en un número importante de aspectos. Casi la mitad de los que contestaron en el sur de Texas era trabajadores agrícolas; en El Paso, casi dos terceras partes de los trabajadores estaban relacionados con la construcción o industria. El ingreso familiar reflejó la diferencia en el nivel de empleo. El ingreso promedio anual de las familias que participaron en la encuesta del sur de Texas fue de \$6,785 anuales (dólares americanos) en comparación con los $\$ 11,497$ de la contraparte en El Paso.

En ambas áreas, más del $95 \%$ de los encuestados eran de origen hispano, y más de un tercio nacieron en México. Más del 70\% de los jefes de familia del sur de Texas y casi una tercera parte de éstos en El Paso no sabían leer o escribir inglés, o no lo hacían bien. Sólo el $16.6 \%$ de los residentes mayores de dieciocho anfos que fueron entrevistados en el sur de Texas habfan terminado la secundaria, en comparación con el $37.9 \%$ de El Paso. El acceso a los servicios de agua potable, drenaje y salud era problemático para la mayoría de las familias de las colonias en ambas áreas. A pesar de las similitudes, parece ser que los residentes de las 
colonias en EI Paso tienen trabajos más estables y mejor remunerados, asf como un nivel de educación formal más alto. Posiblemente esto se deba a que, durante la década de los ochenta, las oportunidades de empleo urbano en El Paso hayan atraido a diferentes tipos de inmigrantes mexicanos que aquellas áreas rurales del sur de Texas.

La población de las colonias en El Paso y sur de Texas tienen un acceso poco satisfactorio en cuanto a la dotación de muchos servicios públicos, esto debido a su aislamiento geográfico y a sus deficiencias en el dominio del inglés. Sin embargo, la mayoría de la atención de los medios de comunicación y los esfuerzos de organización de la población local, se han centrado en el aprovisionamiento adecuado de agua y del sistema de alcantarillado a las decenas de miles de personas que ven amenzada no sólo su propia salud, sino la de toda la comunidad. El problema del agua potable en el sur de Texas no parece ser tan agudo como en el municipio de El Paso; el Consejo para el Desarrollo Acuífero de Texas reportó en 1988 que el $86 \%$ de los habitantes de las colonias estaban conectados a una abastecedor de agua autorizado (U.S. Congress, 1988:54). En el valle bajo de el municipio de El Paso, así como en otras áreas fuera de los límites de la ciudad, las conexiones de agua no están disponibles. El Consejo de Servicios Públicos de la ciudad de El Paso (Public Service Boar of the City of El Paso) finiquitó este servicio en 1979, "aduciendo razones económicas y de planeación". Según John Hickerson, el entonces director del Consejo de Servicios Públicos, dijo: "los fraccionamientos ilegales se estaban saliendo del control"; no obstante esta postura de las autoridades de la ciudad, entre 1979 y 1988, fueron aprobadas por el municipio de El Paso aproximadamente 160 nuevos fraccionamientos sin abastecimiento de agua (U.S. Congress, 1988:146).

Irónicamente, el agua de manantial abunda en el área de Socorro-San Elizario, donde la mayoría de esos nuevos fraccionamientos fueron desarrollados; debido a su proximidad con el Río Grande, en algunos lugares el agua de manantial puede ser obtenida a tres pies de profundidad (aproximadamente un metro). Así, casi todas las familias de estas áreas tienen acceso a agua corriente. Sin embargo, el problema es que las aguas negras contaminan los manantiales. Virtualmente, ninguna de las familias en las colonias en El Paso o en las demás del sur de Texas, están conectadas a sistemas de tratamiento de aguas negras. Es más frecuente que los sistemas alternativos - fosas sépticas, letrinas y retretes fuera de la vivienda- estén deficientemente construidos que lo contrario.

De acuerdo con el Dr. Laurence Nickey, director de Salud de la Cabecera Municipal en El Paso (City-County Health District) "toda el agua de manantial está potencialmente contaminada" (U.S. Congress, 
1988:37). El problema de la contaminación está empeorando en ambas áreas, pero especialmente en el sur de Texas por los residuos de fertilizantes, pesticidas y sales (U.S. Congress, 1988:504). La encuesta de los Servicios Humanos en Texas indica que, aparentemente, los residentes conocen los peligros de la contaminación del agua de los pozos, porque menos del $6 \%$ de las familias la beben. No obstante, una proporción mucho mayor la utiliza para lavar y cocinar (Texas Dept. of Human Services, 1988) y un gran número de personas almacenan el agua de los camiones cisterna en recipientes contaminados o en condiciones poco seguras.

Si bien no se han hecho estudios que directamente vinculen el agua con las enfermedades en las colonias texanas, las estadísticas de salud denotan un panorama poco alagüeño. En 1986, las tasas de salmonellosis y hepatitis de la población de El Paso fueron cuatro y tres veces superiores a la tasa nacional, respectivamente. Además, en el municipio hay más casos de tuberculosis que en el total de diecinueve estados (El Paso Herald-Post, 7 de abril de 1987).

Un sondeo en materia de salud, efectuado en 1988 en San Elizario por el Centro de Ciencias de la Salud de la Universidad de Texas, reveló que una tercera parte de los niños de ocho años que se incluyeron en la encuesta tenían anticuerpos de hepatitis del tipo A; el cuadro se incrementó al $90 \%$ en los encuestados de 30-34 años de edad (Sawyer and Brown, s/f :5). Es difícil asegurar que el agua en malas condiciones sea la principal causa de las condiciones de salud en el municipio de El Paso y el sur de Texas; la proximidad con México, la educación limitada y los bajos ingresos, probablemente son factores importantes que contribuyen a esta situación. No obstante, las autoridades en salud y el público en general temen la posibilidad de que las epidemias originadas en las colonias se hayan incrementado.

\section{CÓMO SE HAN DESARROLLADO LAS COLONIAS: EL CASO DEL MUNICIPIO DE EL PASO}

El comportamiento de los asentamientos de tipo colonia en el municipio de El Paso, probablemente no sea comparable con desarrollos similares en el resto del sur de Texas, Caléxico, California o cualquier otra región del lado norteamericano de la frontera; la dinámica de los asentamientos irregulares en la franja fronteriza ha sido poco estudiada e imperfectamente entendida. Sin embargo, a pesar de las diferencias en la estructura económica regional, grado de urbanización y localización geográfica, el crecimiento de las colonias de mayor tamaño en esta década, a lo largo de la frontera México-Estados Unidos, ha estado influenciado por el incremento 
de la inmigración mexicana en las comunidades fronterizas de Estados Unidos, y por el déficit existente de viviendas accesibles. Bajo esta perspectiva, las observaciones preliminares acerca de la experiencia de El Paso quizá sean ilustrativas para otras comunidades fronterizas.

Los mexicanos de bajos ingresos recién llegados, por años buscaron en la zona sur de El Paso su primer refugio ${ }^{5}$. El subdesarrollo de esta área segregó y aisló a sus residentes, quienes comprenden un conjunto de fuerza de trabajo barata no calificada para los negocios y familias locales, y presentan las peores condiciones de vivienda, salud y criminalidad en la ciudad (García, 1981:135). Para 1910, la mayoría de los anglos ${ }^{6}$ había huido del área (Marquez, 1985:54), pero antes de los sesenta, cerca de una cuarta parte de la población de la ciudad vivía en vecindades de la zona sur, muchas de las cuales desafían su descripción. Desde inicios del siglo, las autoridades anglas de la ciudad y los líderes de la comunidad han condenado la existencia de vecindades densamente pobladas del centro de El Paso; la mayoría de ellas carecen de ventilación, plomería, agua corriente y sistema de recolección de basura adecuados. Empero, la prolongada ausencia de los propietarios de las vecindades de esta deteriorada zona fue una poderosa fuerza para el mantenimiento del status quo. Aún más importante, los mexicanos recién llegados, que mantuvieron la mano de obra barata de la economía regional, no tenían acceso a vivienda en ningún otro lugar.

Durante las décadas de los sesenta y setenta, el Tratado de El Chamizal, la erradicación de los barrios bajos, los programas federales en apoyo a la vivienda y de nuevo los intereses en los proyectos comerciales en la zona sur, finalmente trajeron un cambio en el barrio hispano de El Paso. En medio de las protestas de los activistas del barrio, el proceso de renovación urbana se tradujo en la destrucción neta de cientos de unidades habitacionales baratas no subsidiadas, y la relocalización, planeada o espontánea, de miles de hispanos de bajos ingresos provenientes de las útiles vecindades densamente pobladas de la zona sur.

Entre 1960 y 1980, el número de unidades habitacionales disponibles se redujo en un 55\%, y la población del área declinó de 28,888 a 11,378, esto es, el $60.6 \%$ (U.S. Census, 1960-1980). Irónicamente, muchas de las familias desalojadas no tenían acceso a las más de 2,000 unidades habitaciones públicas que fueron construidas fuera del barrio. El problema de la

5 La zona sur de El Paso generalmente se refiere al segundo barrio, el cual mide 4 millas cuadradas y se ubica al sur del centro principal de la ciudad (central business district) y se extiende hasta la frontera con México.

6 N. del T. El término "anglo" se utiliza en Estados Unidos para referirse a la población blanca que es nativa de ese país, quienes no son descendientes de mexicanos. 
vivienda de las familias más pobres fue exacerbado por las campañas periódicas tendientes a reforzar los códigos de construcción y a rehabilitar las vecindades existentes, lo cual condujo a un alza de las rentas de las viviendas más accesibles de la ciudad.

Las acciones federales y locales que afectaron la zona sur, en pocas ocasiones consideraron las necesidades de vivienda de esa población. En 1940, se inició la construcción de una carretera (Paisano Drive) en el sur de El Paso que físicamente aisló el barrio del resto de la ciudad; estimándose que 6,000 personas fueron desplazadas por el proyecto (Marquez, 1985: 65). A su vez, la ubicación fronteriza del área la hizo vulnerable ante las políticas internacionales. En 1963, cuando Estados Unidos devolvió varios cientos de acres a México de acuerdo al Tratado de El Chamizal, 5,600 residentes fueron removidos de la zona sur. Menos del $2 \%$ de las personas desalojadas fueron reubicadas en viviendas públicas y muchos permanecieron en el barrio en los departamentos de amigos o parientes (Marquez, 1985: 91-92). A pesar de que El Tratado de El Chamizal contempló las compesaciones a los dueños de las propiedades afectadas de la zona sur, menos de $15 \%$ de los habitantes de dicha zona eran propietarios de los predios que ocupaban (U.S. Census, 1960).

Dos de los asentamientos más antiguos del municipio, clasificables como colonias: Sparks Addition y Moon City, crecieron justo al este de los límites de la ciudad, como resultado directo del Tratado de El Chamizal. Sparks Addition fue iniciado en 1959 y, eventualmente, fueron vendidos 1,400 predios residenciales. Este asentamiento está ubicado en medio de las dunas de arena del desierto y, no obstante de que la mayoría de los residentes tuvo acceso a la energía eléctrica en principios de los ochenta, aún carecen de agua y servicios sanitarios. El agua tiene que ser llevada en camiones cisterna y almacenada en depósitos que frecuentemente están contaminados, y la mayoría de las familias todavía cuenta con letrinas fuera de la casa (El Paso Times, 18 de septiembre de 1980).

La ciudad de El Paso empezó a solicitar ayuda en materia de vivienda al departamento de Vivienda y Desarrollo Urbano a principios de la década de los sesenta; sin embargo, casi no hubo avances hasta 1970, cuando el departamento mencionado anunció la autorización de 80 millones de dólares para la rehabilitación de viviendas (Marquez, 1985: 125). Últimamente, la mayoría de estos fondos furon utilizados en la construcción de 3,400 unidades habitacionales del estado, pero de éstas sólo 443 fueron construidas en la zona sur (Marquez, 1985:143); el resto fue distribuido en toda la ciudad. Durante este periodo de construcción de vivienda del estado (1970-1975), la administración del alcalde Fred Hervey llevó a cabo lo que después se conoció como el Programa de Erradicación de Barrios Bajos 
(Slum Eradication Program). Aduciendo el fracaso de los propietarios do los vecindarios para elevar las condiciones de sus edificios al estándar do vivienda de la localidad, las autoridades de la ciudad ordenaron la destrucción de 68 edificios habitacionales donde vivían casi 3,000 personas; otros 57 vecindarios y residencias unifamiliares fueron destruidos por sus propietarios, quienes aparentemente se anticiparon a dichas acciones. Cerca de la mitad de las personas desalojadas fueron ubicadas en viviendas del estado. Así, el resultado real de las acciones fe- derales y del gobierno local no fue la mejora o el aumento de las viviendas disponibles en el barrio, sino la destrucción parcial de la comunidad y la dispersión de la mayoría de sus residentes (Marquez, 1985 :161-167).

Las dificultades para mantener una disponibilidad adecuada de vivienda para familias de bajos ingresos en una frontera permeable, entre la nación más rica del mundo y un país populoso en vías de desarrollo, rápidamente se hicieron evidentes, antes de que la década de la "renovación urbana" terminara en El Paso. Tan pronto que, en 1975, el Departamento de Planeación de la Ciudad fue alertado acerca de escasez de vivienda a precios modestos. Uno de sus estudios estimó que el $70 \%$ de todas las personas que deseaban comprar una casa en El Paso no estaban en posibilidades de hacerlo (Dept. of Planning..., 1975:15). El programa para erradicar los barrios bajos aparentemente empeoró las condiciones de vivienda en la zona sur de aquéllos quienes no pudieron dejarla o no quisieron hacinar los departamentos y casas de sus familiares (El Paso Times. 24 de abril de 1975). El incremento del número de unidades habitacionales estatales fue insuficiente, incluso para aquellos que reunían los requisitos. Los mexicanos que estaban en calidad de ilegales en la zona sur, en algunos casos el 50\% o más en un barrio, no tuvieron acceso a la vivienda subsidiada. Así, a finales de los setenta la lista de espera para las unidades existentes era aproximadamente de 2,000 solicitudes; y las autoridades en vivienda periódicamente suspendían la entrega de solicitudes para no crear falsas espectativas entre las familias de bajos ingresos. Para 1977, la situación en materia de vivienda se había convertido tan crítica y una cuestión tan delicada que, el entonces alcalde, Ray Salazar, declaró (refiriéndose a la zona sur) que: "Uno de estos días va a estallar y tendremos alli otro Watts" (El paso Herald Post, 10 de mayo de 1985).

La zona sur no estalló, a pesar de que, de hecho, los fondos para subsidiar la vivienda disminuyeron drásticamente durante los ocho años de la administración de Reagan (Time, 24 de octubre de 1988). Actualmen-

7 N. del T. Watts es una sección de Los Angeles. Durante la década de los sesenta, la población negra de este lugar estuvo varios días amotinada. 
te, veinte o menos unidades habitacionales subsidiadas en El Paso están siendo agregadas anualmente al inventario de viviendas estatales; $\sin$ embargo, otras presiones adicionales surgieron en el mercado local de viviendas, que fueron generadas por un número aún no determinado de inmigrantes mexicanos, legales e ilegales, quienes huyeron de la prologanda crisis económica mexicana o quienes respondieron a las oportunidades presentadas por la nueva ley de inmigración. La válvula de escape de los hispanos de escasos recursos han sido las colonias. Los agricultores de la parte baja del valle, ante las pocas utilidades generadas por las cosechas como el algodón, respondieron con el fraccionamiento y desarrollo de 2 mil acres de tierras de cultivo para ser usada con fines residenciales; ellos dieron respuesta a la acorralada demanda de vivienda de las familias de bajos ingresos mediante la venta de lotes de medio acre de superficie (aproximadamente doscientos metros cuadrados), con tan sólo \$100 dólares de anticipo y mensualidades de \$75. De esta manera, entre 1970 y 1980 , la población de las comunidades rurales de la parte baja del valle como Socorro y San Elizario, se incrementó de 3,660 a 10,780, es decir casi el $200 \%$. Durante este mismo periodo, la proporción de la población que habla vivido en la ciudad de El Paso cinco años antes aumentó de 2.6\% a $18.0 \%$, y la proporción de residentes nacidos en el extranjero se incrementó de $8.0 \%$ al 31.4\% (U.S. Census, 1970, 1980). Esta población parecer ser que ha crecido aún más rápidamente después de 1980.

La ley texana otorga a las autoridades municipales poco control sobre cl desarrollo de áreas no incorporadas; puede señalar el tamafio mínimo de los lotes de los fraccionamientos nuevos, bajolo previsto en los cstatutos de salud del estado, pero las atribuciones del municipio con mucho terminan ahí. Las leyes del estado permiten que las ciudades de tamaño como El Paso establezcan jurisdicciones extraterritoriales (JET) hasta de cinco millas fuera de los límites establecidos de la ciudad. Por tanto, la ciudad puede ampliar su jurisdicción hasta las JET, bajo el supuesto de que con ello preparará mejor aquellas áreas para eventuales anexiones legales. El Paso amplió sus límites en 1974, adquiriendo autoridad sobre lo que actualmente es un área de 300 millas cuadradas de territorio municipal no incorporado. Parad6jicamente, la ciudad prefirió no ejercer su autoridad y el ayuntamiento de la ciudad no autorizó recursos para la legalización.

En 1978, las autoridades municipales se quejaron públicamente de los "barrios bajos", que rápidamente estaban absorbiendo las antes áreas rurales, culpando a las autoridades de la ciudad por haber eludido sus responsabilidades con respecto a las JET (El Paso Times. 31 de mayo. 1978).

A principios de la década de los ochenta, la Comisión de Planeación de El Paso identifico 102 fraccionamientos en las JET que no habían sido 
registrados y aprobados por las autoridades de la ciudad, y cuyo desarrollo violaba las disposiciones en materia de zonificación; cuarenta y dos dc ellos estaban en la mira para algún tipo de acción legal. En la mayoría de los casos, las propiedades rurales simplemente han sido subdivididas en lotes, y vendidas mediante contratos de compra-venta sin que existieran planes para la construcción de caminos, líneas para la introducción de servicios públicos o tuberías de agua (El Paso Herald Post. 10 de mayo de 1985). En ese entonces las autoridades de la ciudad estaban renuentes a considerar la anexión de esas áreas que estaban dentro de las JET; según el alcalde Jonathan Rogers, "A medida que la ciudad da pasos hacia la anexión, heredamos un tremendo desorden que virtualmente es imposible de limpiar" (El Paso Herald Post. 21 de febrero de 1984); a pesar del abandono de sus responsabilidades por parte de las autoridades, la comunidad ha encontrado una solución temporal a su ecasez de vivienda, pero a costa del municipio, y quizá también de su crecimiento futuro y bienestar.

\section{ALTERNATIVAS PARA EL FUTURO}

Este trabajo ha argumentado que la proliferación de asentamientos irregulares, en áreas no incorporadas del lado norteamericano de la frontera México-Estados Unidos, es el resultado de los bajos ingresos y la severa falta de viviendas para familias de escasos recursos. Dicha falta empeoro en las décadas de los setenta y ochenta en ciudades como El Paso, por la destrucción de los vecindarios que eran propiedad de particulares, por la virtual desaparición de los apoyos federales para la construcción de nuevas viviendas estatales y por un aparente incremento del número de familias mexicanas que prefieren vivir en el territorio fronterizo estadounidense.

Las autoridades públicas locales carecen de la información, recursos, espacios de jurisdicción y voluntad para manejar el rápido crecimiento de las colonias. Por omisión, adoptan una política de no intervención, particularmente en el caso del municipio de El Paso, mientras que las demandas de vivienda fueron satisfechas a través del desarrollo y venta de lotes fraccionados en áreas rurales que tienen pocos - o carecen de-servicios públicos e infraestructura. Irónicamente, si a las personas que fomentaron esos fraccionamientos se les hubiera exigido la introducción de servicios básicos como agua, conexiones sanitarias y accesos pavimentados, debido al incremento de costos, muchas menos unidades habitacionales hubieran sido agregadas al inventario de viviendas de familias de escasos recursos en la frontera. Así como son de caóticas, feas y antihigiénicas, muchas de 
las colonias, representan la mejor solución para las posibilidades de sus residentes ante un problema que el gobierno, a todos los niveles, no podía ni quería afrontar.

La política de no intervención ya no es posible, debido al evidente número de personas que ahora residen en colonias y a la ampliamente difundida publicidad que han recibido. Casi todas las alternativas políticas para mejorar las condiciones de vida en estos barrios, se han centrado en dar a sus residentes acceso a sistemas de agua y servicios sanitarios comunes; los costos de tales programas son muy variables, y ni las familias de las colonias ni la sociedad local disponen de los recursos necesarios (U.S.Congress, 1988). Si tales mejoras deben ser efectuadas en gran escala en un periodo breve, deberá existir una disponibilidad de recursos asignados por el gobierno federal o estatal, o por ambos.

En ese sentido, al menos existen dos fallas graves. Primero, mientras que los gobiernos federal y estatal han expresado mucho interés en los problemas de las colonias, no han habido grandes asignaciones que otorguen los recursos para su solución ni parece que habrán en el futuro cercano, dada las limitaciones presupuestales que han enfrentado la administración de Bush y el gobierno local; y segundo, cualquier programa eficaz debe considerar una subvención, porque muchas familias simplemente no pueden afrontar los costos de conexión. Más importante, el agua y las conexiones sanitarias para los habitantes actuales de las colonias no se dirigen al problema básico - la vivienda-. Muchos proyectos de construcción de vivienda para familias de bajos ingresos están en camino en ambos lugares, el sur de Texas y municipio de El Paso, pero no son del tamaño y no tiene apoyos financieros para enfrentar el problema. La demanda de vivienda barata continuará creciendo, ఒcuánto? dependerá de la tasa de inmigración de mexicanos de escasos recursos hacia la región fronteriza, de la tasa crecimento natural de los residentes hispanos pobres, y del incremento del ingreso; factores que los planificadores no pueden controlar.

El hecho de que las colonias, a todo lo largo de la frontera, estén saturadas de descendientes de mexicanos es una fuente potencial de problemas sociales y de una situación política explosiva. Las autoridades de la ciudad y del municipio en Texas, claramente parecen favorecer los intereses de un pequeño grupo de urbanizadores por encima del bienestar de decenas de miles de hispanos, cuyas alternativas de vivienda están severamente limitadas y quienes tienen poco acceso a procesos políticos e institucionales.

La creación y crecimiento de áreas residenciales marginales, en las que se ha negado a la gente el acceso a los servicios mínimos de salud, 
educación, transporte y otros servicios públicos, puede ser interpretadı como el resultado de políticas racial o étnicamente parciales por parte do las autoridades locales y estatales. Ya sea que los problemas de las coloniux sean el resultado de la discriminación, avaricia, o simple negligencia o incompetencia de las autoridades públicas, el mantenimiento del status quo amenazará el futuro bienestar de las comunidades fronterizas como EI Paso. De manera creciente, mucha de la fuerza de trabajo de las ciudaden fronterizas tendrá que ser sacada de estas áreas no incorporadas que van en aumento, y la productividad, ingreso y habilidades de esos trabajadorew será la principal fuerza que modele el desarrollo económico de la región.

Una propuesta más realista sería planear y regular los futuros fraccionamientos de tipo colonia. Esto no significa que se prohíba el desarrollo de asentamientos para familias de escasos recursos en áreas rurales a to largo de la frontera, ni que a los urbanizadores de futuras colonias se low exija la dotación de la infraestructura que está considerada para la mayorfa de las familias estadounidenses; cualquiera de esas medidas agravaria ol problema de la vivienda. A esas familias que tienen recursos y alternativas de vivienda limitados, se les debería permitir comprar un terreno, si esta disponible, y construir su hogar "con el sudor de su frente"; sin embargo, los municipios deben de asegurarse de que los residentes, agua y sistema de drenaje se sujeten a los estándares mínimos de salubridad, a fin do proteger a la comunidad en su conjunto. Algunas personas ya están experimentando mecanismos alternativos como autopurificación de aguu y alcantarillados, que bien pudieran ser instalados y mantenidos por los usuarios y que son accesibles para la mayoría de los residentes de las colonias. Exigir tales sistemas llena los requisitos mínimos que se traducirían en un incremento del costo de mudanza hacia las colonias, quizA suficiente para detener en algo la tasa de inmigración de mexicanos, pero no suficiente para acabar con el incremento de viviendas baratas. Unu solución de este tipo - la cual principalmente depende de los recursos, planificación y regulaciones locales_-, está basada en la realidad de tener que enfrentarse a los procesos demográficos de finales del siglo veinte sin los significantes apoyos financieros de Washington. 


\section{BIBLIOGRAFÍA}

COPELAND, Claudia y Corpas, Mira. 1987. Border State "Colonias": Background and Options for Federal Assistance. Congressional Research Service. Washington, D.C.

DEPARTMENT OF PLANNING, RESEARCH, AND DEVELOPMENT, CITY OF EL PASO, TEXAS. 1975. El Paso:Transition in Housing. El Paso, Texas. Septiembre de 1975.

GARCIA, Mario T. 1981. Desert Inmigrants. New Haven: Yale University Press.

MARQUEZ, Benjamín. 1985. Power and Politics in a Chicano Barrio. Lanham, MD: University Press of America.

SAWYER, John y Brown, John. S/F. "San Elizario Health Survey Final Report". University of Texas Health Science Center. San Antonio, Texas (mimeo).

TEXAS DEPARTMENT OF HUMAN SERVICES. 1988. "The Colonia Factbook". Austin, Texas. Junio de 1988.

U.S. CENSUS. 1960, 1970 y 1980.

U.S. CONGRESS, HOUSE, COMMITTEE ON PUBLIC WORKS AND TRASPORTATION. 1988. "Inadequate Water Supply and Sewage Disposal Facilities Associated With 'Colonias' Along the United States and Mexican Border, Hearings before a subcommittee on Water Resources." Segunda sesión del centésimo Congreso.

\section{PERIODICOS Y REVISTAS}

\section{EL PASO HERALD-POST}

- "El Paso's 10,000". 7 de abril de 1987.

- "28,000 Lack Public Water, Sewer Lines". 24 de nov. 1987.

- "City Views Urban Renewal Plan for South EP". 14 de julio de 1977.

- "City Cracks Down On Subdivisions Causing Havoc". 10 de mayo 1985.

- "El Paso Targets Illegal Subdivisions". 21 de febrero 1984.

EL PASO TIMES.

- "Sparks Residents Reach Dead End With Developer". 18 de sept. de 1980.

- "Finding Paint Grim Picture of Quality of Life In Subdivision". 20 de marzo de 1986. 
- "Preserving the Barrio: A Trauma for Families and EP Officials". 24 de abril de 1975.

— "EP City Limits Create Disorderly Problem". 31 de mayo 1978.

TIME

— "The Homeless: Brick by Brick". 24 de octubre de 1988.

WASHINGTON POST

- "El Paso's Perimeter of Poverty". 17 de agosto de 1987. 